\title{
Atmospheric Monitoring Strategy for Ground Testing of Closed Ecological Life Support Systems
}

\author{
John Feighery \\ NASA Johnson Space Center \\ Ivan Cavenall, Amanda Knight \\ Lockheed Martin
}

Copyright @ 2004 SAE Intemational

\begin{abstract}
This paper reviews the evolution and current state of atmospheric monitoring on the International Space Station to provide context from which we can imagine a more advanced and integrated system. The unique environmental hazards of human space flight are identified and categorized into groups, taking into consideration the time required for the hazard to become a threat to human health or performance. The key functions of a comprehensive monitoring strategy for a closed ecological life support system are derived from past experience and a survey of currently available technologies for monitoring air quality.
\end{abstract}

Finally, a system architecture is developed incorporating the lessons learned from ISS and other analogous closed life support systems. The paper concludes by presenting recommendations on how to proceed with requirements definition and conceptual design of an air monitoring system for exploration missions.

\section{INTRODUCTION}

This study began as a response to a request to identify the integration issues that arise in an advanced closed loop life support system test that have significant impacts on the integrated system. By asking these questions early in the planning for the Advanced Integration Matrix (AIM), a ground test facility intended to help solve the problems associated with keeping humans alive during long duration space missions, a brief window of opportunity is provided to incorporate the lessons learned from previous experiences on the ground and in space flight without the imposed constraints that come into play once the project is underway.

The evolution of life support technologies to support long-duration space flight created artificial ecosystems for the express purpose of supporting human occupants. As with the natural ecosystems that support life on earth, these artificial systems depend on complex processes and energy input to maintain an otherwise unstable balance. The systems differ in that spacecraft systems contain much smaller buffers for air and water; hence, they afford little opportunity to cope with sudden changes such as a build up of harmful pollutants or a failure of a particular subsystem. Therefore, to ensure that crewmembers are protected from the inherent risks of a closed system, monitoring of the environment is required to both track system performance and alert the crew or ground team to unexpected health hazards.

The information and discussion presented in this paper is intended to serve as a starting point to begin considering the requirements and architecture for an air monitoring system capable of meeting the needs of a long duration planetary mission. These needs require further definition, but some will not be apparent until very late in the design of a vehicle or habitat, when technical problems must sometimes be resolved by accepting the risk of a toxic compound onboard. Therefore, a monitoring system must be flexible enough to accommodate a broad range of potential pollutants and concentrations.

\section{REQUIREMENTS DEVELOPMENT PROCESS}

The first step in developing requirements is to identify the stakeholders who have an interest in the functional capabilities of the system. For a spacecraft air monitoring system, the primary stakeholders include:

ㄱ Toxicologist

- Environmental control and life support system engineers

- Flight surgeon / medical establishment

- Scientific and medical research community

To better understand how requirements for individual compounds came into being, it is helpful to group them according to a few driving principles for monitoring the environment. The following drivers are applicable to both ISS and future missions:

General hazards posed by closed environmental systems. These hazards are due to the artificial 
ecology present in a closed or partially closed life support system. Off gassing from materials and lubricants are typical sources, as well as human or microbial metabolic products.

$\square$ Monitoring life support systems. Monitoring of life support processes enables fault detection, informs routine maintenance decisions about when to replace or repair components, and in some cases is used as feedback control for certain processes.

Unique hazards posed by system design or operations. Often hazardous materials are permitted on the spacecraft when the benefits to the system outweigh the risk posed by a release. To ensure that any unexpected releases are detected before they pose a threat to crew health, routine broad-spectrum monitoring is necessary.

The maximum permissible concentration of a compound will depend on the requirement driver, the toxicity of the compound, the amount of data available in the scientific literature, and the time to effect. These limits are currently set for NASA through a collaborative process involving the National Research Council and other experts, resulting in a list of Spacecraft Maximum Allowable Concentrations (SMACs).

Current ISS air quality requirements are specified in a joint US/Russian document called the Medical Operations Requirements Document, SSP 50260 (Revision B). Since similar compounds can have a cumulative effect on the human body, methods are prescribed for determining the toxicity of groups of compounds. These methods are described in Russian State Standard GOST R 50804-95 and the NASA SMAC Document, JSC 20584.

\section{ISS AIR MONITORING SYSTEM DESCRIPTION AND LESSONS LEARNED}

The International Space Station Air Monitoring system came about through a long process of evolution influenced by technical, financial, political, and international factors. An early split that occurred in the air monitoring system was essentially geographical. NASA's Marshall Space Flight Center (MSFC) was assigned responsibility for design of the life support systems. Once Space Station Freedom became Alpha the new design required that during the first phase of assembly the Russian side was the primary provider of life support systems. Throughout these changes, the responsibility for protecting crew health continued to remain at the Johnson Space Center. Therefore, three very different organizations were now required to work together to adequately monitor and control air quality. As a result, each organization provides unique, independent hardware capabilities that together form the ISS monitoring system.

SNAPSHOT OF CURRENT ISS HARDWARE - The current "gold standard" for environmental verification on ISS is laboratory analysis of returned samples. Both the
US and Russian partners have capabilities (see Table 1) to return samples for analysis using traditional lab techniques that routinely detect a number of common pollutants (James et al, 2001).

Ground monitoring is effective in detecting a wide range of stable compounds collected at a single point in time, but to verify that the environment is safe over a period of 6 month, toxicologists need regular insight into the composition of the atmosphere that is simply not possible when a limited number of samples are returned every $3-6$ months. In addition, the capability to respond to unexpected contamination events is necessary to make informed decisions about whether the crew should remain onboard or make a hasty return to the ground. It is the ultimate goal of the ISS to develop and validate inflight analytical capabilities so that reliance upon sample return is reduced and to pave the way toward longduration exploration missions. Current onboard and archival instruments are listed in Table 1.

MONITORING CAPABILITIES - Stating the names of compounds detected only provides a glimpse of what a robust environmental monitoring program looks like. To provide a better understanding of current ISS capabilities, it is helpful to place all the components together on the same scale (a logarithmic scale is necessary) and then place the compounds into functional groups, which are roughly defined by the following factors:

Typical concentration (or the concentration expected during a contingency event)

$\square$ Time to effect, either through a low or high concentration

- Toxicological priority (based on previous experience, presence on ISS and likelihood of release, impact on human health and performance)

Figure 1 represents an integrated picture of the ISS onboard air monitoring system. For each compound, the orange triangles represent the SMAC 180 day limit and the book-ended bars indicate the detection capability of the relevant monitoring instrument. Approximate boundaries are shown for each instrument to better understand each individual contribution and identify gaps in coverage.

\section{LESSONS LEARNED FROM ISS OPERATIONS}

Hardware Design

1. Onboard Validation and Calibration Standards. Once a complex analytical instrument is designed and built to measure compounds in the parts per billion range, how do we know it is working once it enters service? The Volatile Organic Analyzer (VOA) was validated by comparing in-flight measurements of common contaminants against archived samples that were taken at the same time. This process took many months and resulted in validation for 15 of the 20 target compounds. This approach will not be 


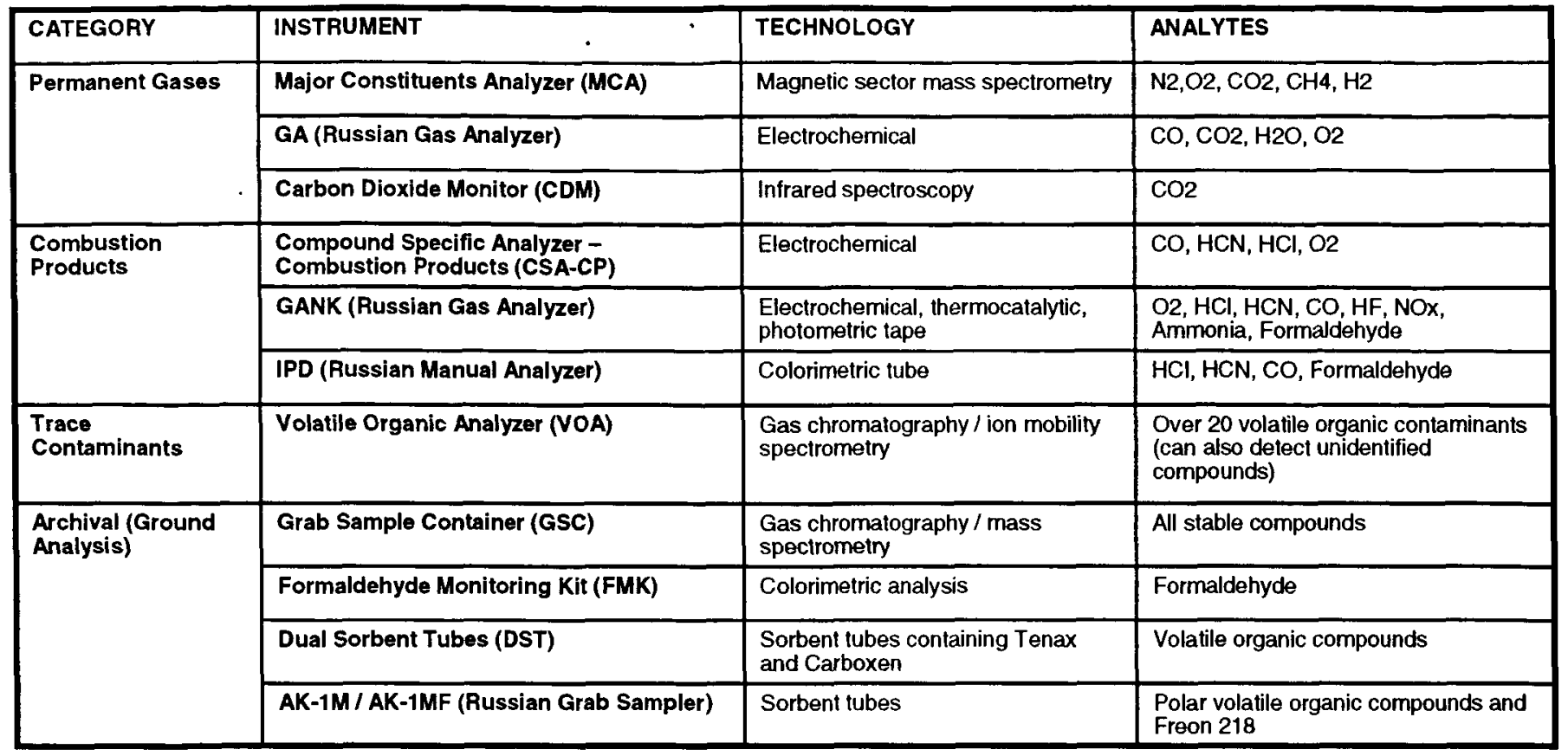

Table 1 ISS atmospheric monitoring instruments

possible for exploration missions, but even if we assume that only well-proven technologies will be used, problems still exist with this method. Many of the compounds that must be monitored should not be present unless a failure has occurred.

Furthermore, when an air quality problem is detected

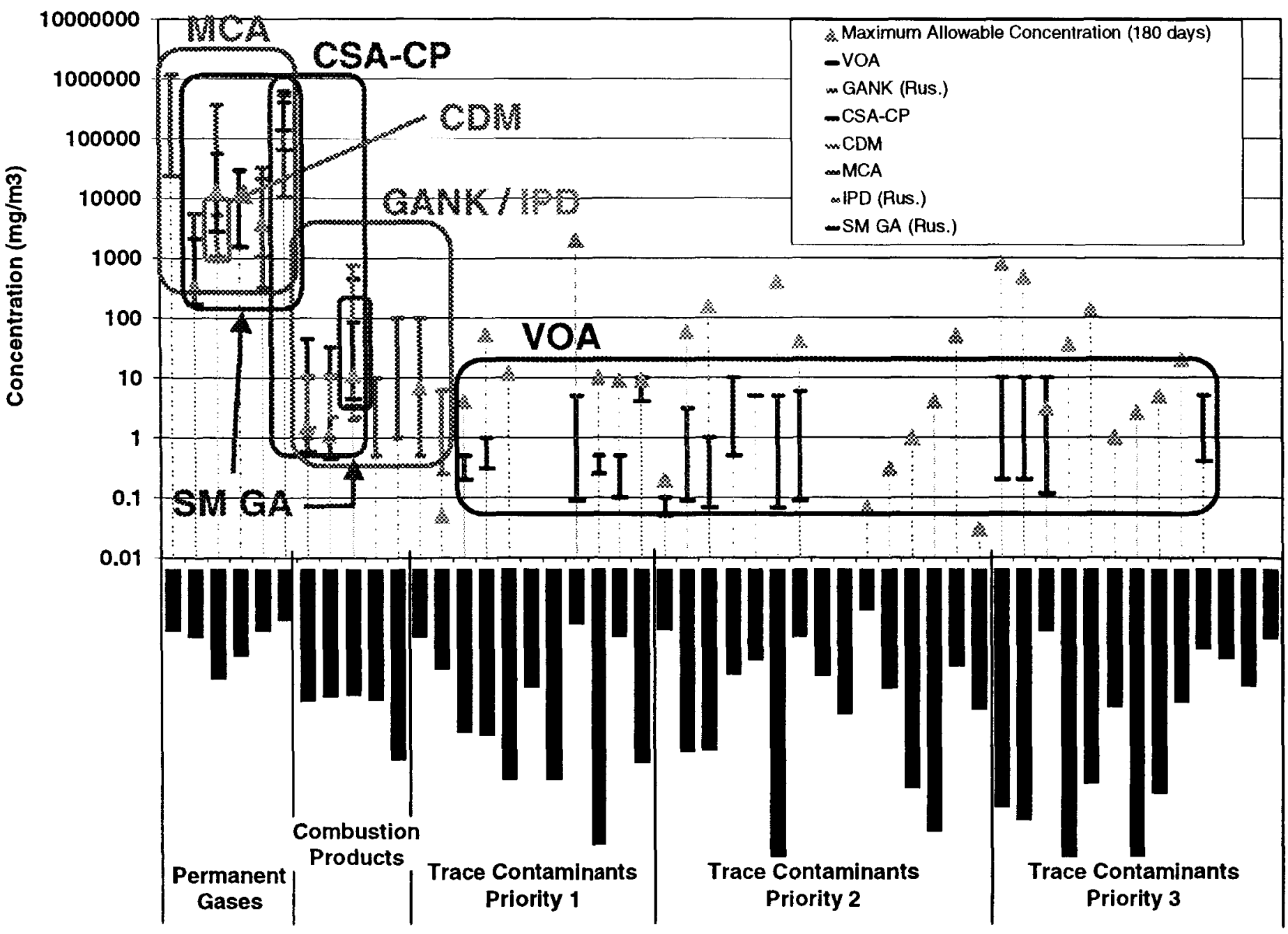


and difficult decisions must be made regarding crew safety, mission managers will need to quickly rule out the possibility of an instrument malfunction.

Providing sample containers or other calibration standards containing a dilute mixture of common contaminants can provide in-flight validation and verify that an instrument is continuing to perform within specifications.

2. Smaller is Better. It goes without saying in the age of Moore's Law that complex electromechanical systems will become smaller and better over time, but nowhere is this principle more enabling than in spacecraft instruments. Consider the case of trace gas analyzers: by decreasing the size of the preconcentrator, GC column, and detector, a number of indirect benefits are realized. The size and number of heaters are both reduced since less heat is lost in transit between the components and the thermal mass is lower. The overall power demand drops, and so does the need for insulation, cooling, and fuses, since there is less heat to deal with both during normal operations and in a potential runaway overheating contingency. Since the total volume of the sample path is reduced, less carrier gas is required and smaller pumps and valves are possible since the pressure and flow demands are less. With recent advances in micro-electromechanical systems, it is possible to envision in the not too distant future a handheld trace gas analyzer capable of meeting most of the requirements for longduration missions. By working toward miniaturization of the air monitoring components, many unforeseen benefits will be realized in both design and operations.

Interfaces With Other Systems

1. Understanding Control and Data Handling Requirements. The means by which the components of the monitoring system interface with the flight command and control system must be consistent with the priority of the information. For example, the Volatile Organic Analyzer (VOA) takes 3 hours to complete a run, then the data must be downlinked for interpretation by experts on the ground. This is an acceptable delay for information that the crew does not need to immediately act upon, and the VOA does not need to be communicating via a real-time data bus. In fact, use of this specialized communication standard caused confusion that resulted in a serious software error, which will be discussed in the next section. By contrast, an instrument such as an electrochemical oxygen sensor could benefit greatly from being placed on a network that constantly commands it to take readings and relay them to the ground or onboard control systems. Data transfer should be prioritized based on how time-sensitive it is to onboard systems and ground experts.

2. The Importance of Integrated Testing. The Volatile Organic Analyzer spent the first year of its operational life plagued by software lockups and reboots that were eventually traced to a line of code in the software interface with the 1553 data bus. A misinterpretation of the 1553 standard resulted in a corrupted array that built up over time and eventually consumed all the processing power of the onboard computer. Some indication of a problem with the software was observed during integrated ground testing with the US Lab Module computer system, however troubleshooting was not possible due to the tight pre-launch schedule. At the time, the VOA team did not have access to a simulator. Once problems were apparent on-orbit, many weeks of runtime with a 1553 simulator were required to pinpoint and fix the error. An investigation pointed toward incorrect interpretation of the software interface documentation, compounded by the lack of opportunity for long-term testing which would have caught the problem before flight. The VOA ran for hundreds of hours on the ground during calibration and acceptance testing, but this testing was not integrated with a flight-like data system. The irregularities that did occur during the Lab integrated test did not have time to build to the point that the onboard computer locked up, so it was concluded based on the data available that they were minor nuisances that could be resolved later. This is one of many examples in the history of space flight illustrating the importance of long-term integrated testing under flight-like conditions.

3. Portable vs. Integrated Equipment. Early in the design phase, careful consideration should be given to the benefits and drawbacks with integrated systems, meaning semi-permanently installed hardware that makes continual use of higher-level resources. VOA is an example of an integrated system: it is installed in payload rack and requires power, data, air cooling, and nitrogen gas from the US Lab. This design was necessary due to the high demand VOA placed on all these resources. Future monitoring equipment can be expected to be smaller, lighter, and less resource intensive. It may be possible to make such equipment portable, with occasional recharging, re-filling, and data downloading. This design not only has the benefit of being portable, but also avoids significant engineering and testing costs associated with use of system-level resources. However, now the instrument requires a battery and charging system along with gas reservoirs, and it must be more rugged. Trade studies may determine that the vehicle still is best served by an integrated system, but this should not be a baseline assumption.

\section{System Architecture}

1. Define Criticality and Failure Scenarios. Most of the current ISS environmental monitoring hardware was designed prior to the actual operation of the ISS or even the Shuttle-Mir Program and reflects the previous Space Shuttle paradigm regarding safety and criticality. All the monitoring systems (except for the combustion products monitor) were designated as Criticality 3, meaning that failure would not cause loss of crew or mission. These instruments monitor for failures of other systems and unknown risks that 
cannot be adequately predicted. The systems they monitor were considered critical because their failure could cause immediate impacts to the mission. By contrast, the failure of a monitoring system causes a slow and steady accumulation of risk due to the lack of insight into the environment. Over time, this accumulated risk can pose a direct threat to the crew and mission because a problem that could have been diagnosed and resolved early can progress into a serious situation by the time crew symptoms or other indirect indications are present. Appropriate definitions of criticality depend on a complete understanding of the system impacts caused by a failure and how this affects overall mission risk. Tools such as operational scenarios and risk analysis can assist designers in this task.

2. Integration with Water Quality. Currently, ISS has separate systems for air and water quality monitoring. In-flight chemical monitoring of water is currently limited to Total Organic Carbon, $\mathrm{pH}$, and conductivity, but there is strong interest in expanding these capabilities to include identification of volatile organic compounds. A number of techniques are available for performing water analysis using a gas analyzer such as headspace analysis or vaporization. In addition, the source of many volatile organic compounds in spacecraft water is the atmosphere, through recovery and processing of condensate into usable water. It may be possible to predict the water concentration of some compounds based on the atmospheric concentration and modeling of the recovery process. Greater integration between air and water analyzers may help reduce the size and complexity of the water monitoring system.

3. One System, Multiple Users. As mentioned earlier, the ISS air monitoring system developed along multiple independent paths, with different users building their own set of hardware. Since none of the parties could reach a comprehensive agreement on priorities or what the minimum complement of hardware should be, it was difficult to see the system impacts of cutting out individual components when cost and schedule trade-offs had to be made. As a result, the current system has a number of gaps that the ISS program is now working to fill. Some of these gaps include real-time ammonia monitoring, portable reduced pressure oxygen monitoring (needed during the pre-breath protocol used before spacewalks), and continuous carbon monoxide monitoring in sleep areas. A new development model is needed to integrate the requirements for exploration systems. An alternative system architecture is presented in the next section of this paper.

\section{AIR QUALITY MONITORING FOR EXPLORATION MISSIONS}

SYSTEM ARCHITECTURE - The development and operational history of the ISS Environmental Monitoring System illustrates the importance of a common vision for air monitoring requirements. Figure 2 depicts a common platform that all users of a future monitoring system might use to integrate their monitoring needs into a set of common system-level requirements. The specific applications that derive from monitoring requirements are often driven by similarities in the time-sensitivity of the data, the need for portability, and the technology available to perform the function, along with other factors. The evolution of the ISS system points toward some natural functional areas: immediate threat monitoring, closed-loop system process control, longterm human/system health monitoring, and broadspectrum analysis vs. single component detection.

Although the system is primarily organized by timesensitivity, this does not imply that less time-critical data is less important, only that the need to act on it is more immediate. The placement of the communication and location categories are intentionally vague, since these details will need to be worked out as the system evolves. The main intention of this approach is to visualize the entire monitoring system in an integrated fashion and to assist mission designers in understanding the factors that may influence how the rest of the vehicle interacts with the system. In addition, a preliminary list of potential technologies is included along with some system-level integration issues that have been identified through the ISS experience.

POTENTIAL AREAS FOR EXPANDED MONITORING By carefully reviewing the ISS experience and considering the unique aspects of an exploration mission, it is possible to identify some topics requiring further research. The most obvious difference between exploration missions and ISS is that environmental samples will not be returned for ground analysis. Additionally, the longer period of isolation gives rise to greater concerns about long-term exposure to trace pollutants. It is also likely that new life support technologies will be in use, including ecological life support systems and increased closure of water and waste loops, which have the potential to introduce new pollutants. Another unique consideration will be surface operations bringing in material such as dust, frozen or condensed volatile organic compounds, and possibly even Martian microbes.

The following is a list of areas that the AIM working group would like to consider for further research and trade studies to determine if technology development is warranted:

1. Byproducts of Ecological Life Support Systems. The definition of ecological life support used here is any system that sets up an artificial ecology for sustaining human life. Some of these systems are present on ISS, but many more will be included in an exploration mission to recycle water, wastes, and breathable air, and most processes are capable of producing volatile byproducts through chemical reactions or microbial metabolism. Often, unexpected byproducts are produced in such small quantities that they are not detected during materials 


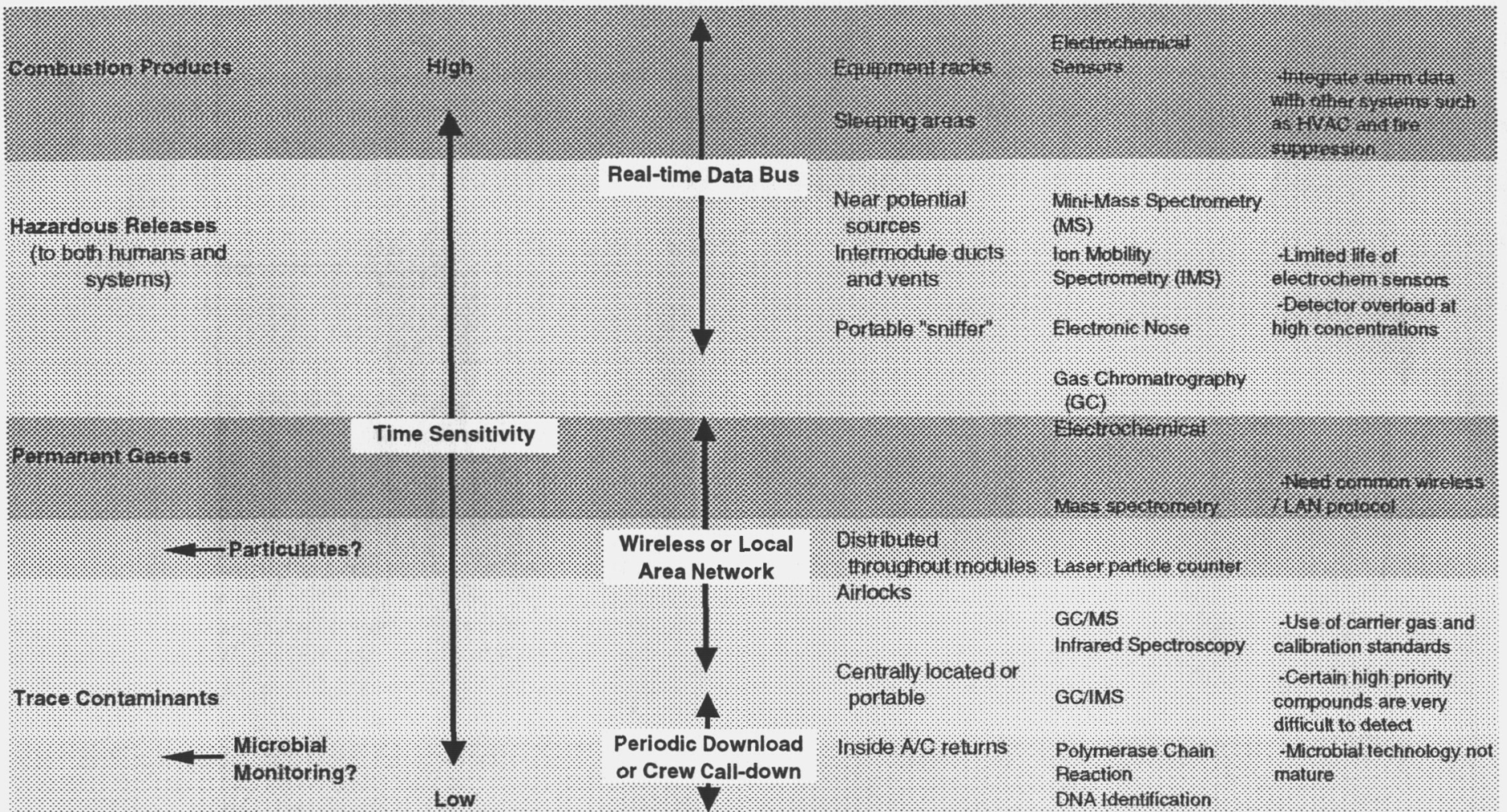

or system off-gas testing. Long-term closed loop testing is the only method to discover these problems before a mission.

2. Airborne Microbes. Microbes that were originally considered benign can become pathogenic over time when humans are isolated from the outside world. Current ISS technology only attempts to quantify total bacterial loads in the air. To study the presence and evolution of airborne microbes over time, microbiologists require frequent sampling and culturing capabilities, either inside the test facility or through analysis of samples in outside labs.

3. Planetary Protection. Another reason to monitor and control airborne microbes is the potential for contamination of the planetary surface. Since one of NASA's primary reasons for exploring other planets is to determine is life is present or was present at some time in the past, care must be taken to avoid contaminating the pristine environment around the spacecraft. Since maintaining a sterile environment is not necessarily practical or even beneficial, it will be helpful to know exactly what strains of bacteria are present onboard the spacecraft to rule out terrestrial sources of any discoveries.

4. Particulate Contamination. Dust will be a constant companion during surface operations on the Moon or Mars, and monitoring may be required to ensure that airborne particulates do not present a threat to human health. Just as trace contaminant limits are set well below levels used by terrestrial regulatory agencies due to unknowns regarding the environment and human immune response in space, particulate limits can be expected to be stringent on a planetary surface.

\section{DEVELOPING AN AIR MONITORING STRATEGY -}

With a comprehensive architecture that integrates all the potential users of the air monitoring system, it is possible to begin work on a plan that will guide specific technology development efforts toward a common goal of supporting long duration human exploration missions. However, this discussion is intended to serve only as a starting point. The next logical step is to establish an Advanced Air Monitoring Working Group that integrates the ISS stakeholders and experience base with the advanced life support community. Such a group could be chartered to develop detailed requirements for different exploration mission scenarios and produce conceptual designs for the various air monitoring systems. Additional recommendations for the group follow.

\section{Recommendations for Advanced Air Monitoring Working Group:}

1. Develop listing of compounds monitored on ISS along with drivers to determine whether they are specific ISS concerns or generic to all closed systems.

2. Research history of air quality requirements and monitoring systems of spacecraft and other analogous closed systems to capture lessons learned.

3. Develop a hazardous/volatile materials tracking database for advanced life support systems and experiments. Any experiment or system proposed should be required to submit inputs. All materials capable of off-gassing or toxic releases should be included such as plastics, electronic components, foams, and lubricants. 
4. Present a potential design for the first AIM Test. Include operational scenarios, resources required, command and data handling, and crew interaction.

5. Identify opportunities for the ISS to be used as a test bed to prove out advanced air monitoring technology and operational concepts.

\section{CONCLUSION}

By working early in the planning phase for exploration missions, a more integrated and efficient atmospheric monitoring system can be developed by considering the overall system architecture and how each component should interact with the crew, ground experts, and other systems. With this framework in place, mission planners and ground test facilities can readily see the impacts of trades and design decisions on crew health and safety. Ground testing of advanced life support systems provides an excellent opportunity to integrate the experience gained through iSS atmospheric monitoring with the unique requirements imposed on exploration missions, with the ultimate goal of providing dependable and effective monitoring equipment to help ensure the success of future missions.

\section{REFERENCES}

1. James, JT, et al (2001) Toxicological Assessment of the International Space Station Atmosphere. Proceedings of the 31st International Conference on Environmental Systems, Orlando, Florida, July 2001 (SAE Paper Number 2001-01-2396).

2. James, JT. (1999) Spacecraft Maximum Allowable Concentrations for Airborne Contaminants, JSC 20584, June 1999 